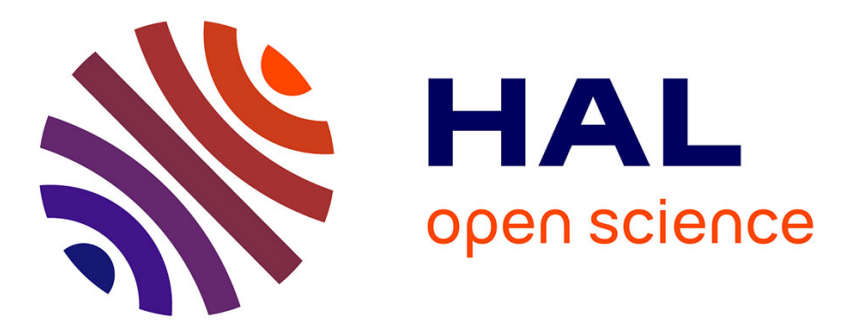

\title{
Programmatic Advertisement and Real Time Bidding Utilization
}

\author{
Dalal A. Alsabeeh, Issam Moghrabi
}

\section{To cite this version:}

Dalal A. Alsabeeh, Issam Moghrabi. Programmatic Advertisement and Real Time Bidding Utilization. 16th Conference on e-Business, e-Services and e-Society (I3E), Nov 2017, Delhi, India. pp.289-297, 10.1007/978-3-319-68557-1_26. hal-01768505

\section{HAL Id: hal-01768505 \\ https://hal.inria.fr/hal-01768505}

Submitted on 17 Apr 2018

HAL is a multi-disciplinary open access archive for the deposit and dissemination of scientific research documents, whether they are published or not. The documents may come from teaching and research institutions in France or abroad, or from public or private research centers.
L'archive ouverte pluridisciplinaire HAL, est destinée au dépôt et à la diffusion de documents scientifiques de niveau recherche, publiés ou non, émanant des établissements d'enseignement et de recherche français ou étrangers, des laboratoires publics ou privés. 


\title{
Programmatic Advertisement and Real Time Bidding Utilization
}

\author{
Dalal A. AlSabeeh, Issam A. R. Moghrabi ${ }^{\text {[0000-0002-4517-7630] }}$ \\ Gulf University for Science and Technology, Kuwait City, Kuwait \\ dalal-alsabeeh@outlook.com, moughrabi.i@gust.edu.kw
}

\begin{abstract}
This paper focuses on the use of Real Time Bidding in Programmatic Advertisement and its utilization with a focus on Kuwait. Real Time Bidding is a new marketing technique that uses information collected from web users and exploits this information in targeting them. This technique, though it saves both time and cost, is neither well known nor utilized in Kuwait. The paper sheds light on some research done on Real Time Bidding and its applications. A two-step study is presented here that aims at testing the efficiency of Real Time Bidding. The first step evaluates the relevance of the used advertisements. The second step uses a survey to assess if the technique may prove effective in Kuwait. Our findings indicate that Real Time Bidding is the closest thing to achieve efficient marketing, yet in order for it to work in Kuwait the technology infrastructure needs to be updated and upgraded in order to achieve the benefits of Programmatic Advertisement.
\end{abstract}

Keywords: Programmatic Advertising, Real-Time Bidding (RTB), Marketing.

\section{Introduction}

Technology is continuously evolving and this makes it much easier to collect more information about the user. For example, cookies stored by visited web sites on the user's drive constitute an information collection mechanism. Having access to such information is very beneficial to not just governments, but also to the marketing agencies. In order for companies to upgrade their profit figures, efficiency in marketing is the key. Efficient marketing means reaching the right audience at the right time, a thing that requires the possession of enough information about the potential customers.

Nowadays, there are countless online advertising platforms. Programmatic advertising is an automated technique that publishers utilize to promote their advertising space to advertisers interested in exploiting that space to reach out to more potential customers. Programmatic advertising promises to improve the targeting of ads, decreasing costs on advertisers and making the web less annoying to users by maximizing the probability of showing them ads that are more likely to be of interest to them [5][9][11]. There are two types of programmatic advertisement the first one is direct 
advertising which is directly buying the advertising slots from the publisher, while the other kind is Real Time Bidding (RTB) which is based on bidding for advertisement spots. This paper focuses on RTB type of Programmatic Advertising, the objective of this paper is to find if RTB works in Kuwait and how acceptable is it.

Prior to RTB, display ads were sold in a reservation contract which is based on a negotiation between the publisher, who is displaying the ad on their website, and the advertiser, who wants to put an ad on that website. After signing on the reservation contract, a Direct Buy is made based on number of ads required, the target audience of those ads and their location [6][13]. An example of a Direct Buy between an advertiser and publisher is advertising specifically for women aged 18-21 who live in $\mathrm{Ku}$ wait. RTB basically is marketers bidding on empty advertisement slots on real-time basis to achieve efficient marketing. Fernandez-Tapia et al. [2] emphasize that this defines a new model in the way digital inventory is purchased: advertisers can buy online inventory through real-time auctions for displaying a banner (or a short video). This model enables advertisers to target individual customers based on their website surfing by using browser cookie information. Based on Google's White Paper Review of the RTB, it is a system that has two intersecting layers, the RTB API and the Real Time Bidder; the "Pipe" and the "Brain". The job of the RTB API the "Pipe" is to provide empty ad slots in a real-time stream to eligible buyers. As soon as an ad slot becomes available for purchase, it is announced. Then, the "Brain" is connected to several "Pipes" which allow them to assess every ad slot announced in order to acquire the best slot for its advertiser. Bidding for advertisement slots, bidders become selective as to which slots they want to buy. That causes them to place premium bids on the audiences that are valuable for the advertiser; the highest bidder gets the slot [3]. Real-Time Bidding enables marketers to customize their ads for each individual. So, basically RTB bundles auctions that ascertain that the premium a bidder is willing to pay is exhausted for every ad impression, in milliseconds. This is certain to work to the advantage of the premium publisher [7]. It can be deduced that the bidder has the upper hand in controlling such a market, but it can also be equally beneficial to the publisher if the demand for their ad space turns out to be high. More than $85 \%$ of marketers believe that Programmatic Advertisement provides more Return on Investment (ROI) than traditional marketing [1].

\section{$2 \quad$ How does it work?}

As programmatic advertising aims to reach the right audience at the right time, it uses online Big Data repositories that contain data on millions of consumers (a good example of such data source are social networking platforms [5]). When an ad is revealed to a web visitor on a particular website (or the ad publisher's site), tracking the visitor's click behavior and, in some cases, whether the click converts to a desired action becomes possible, such as a sale or a sign-up [7]. This platform also has analytical software to excavate the targeted consumers and online shoppers in the database. Machine learning techniques are employed to test out combinations of consumer characteristics that optimize the chance of a purchase resulting from exposure to an ad 
[8] [12]. The inventory (ads) can be bought programmatically and may also be bought algorithmically by unit, in the hope of achieving the intended goal of programmatic advertisement of being an efficient marketing tool. This technology requires two sides, the supply side and the demand side. The supply side, which is the publisher, connects to a virtual ad exchange marketplace to offer each ad slot separately and start an auction for each slot. On the other side lies the ad trading desk which is a demand side platform where it receives an auction request, user information, type of website and the bid level that best matches their marketing strategy. Once all bids are processed in the system, the bidder with the highest bidding price gets the ad slot and then pays the price depending on the auction type. The entire process, from the user entering the website to displaying the banner, consumes around 100 milliseconds [2]. Google explains that the first layer of the "Pipe" which is the RTB API, is available for use, but needs the second layer which is the "Brain" which is the bidder to connect to the "Pipe". Not many companies can afford the acquisition of the technology for building one. So, many of them are having contracts with ad exchange platforms in order to utilize ad slots. It also suggests that there are three ways to do Real-Time Bidding: First, a partner with an ad network that does real time buying on its own inventory that is connected to an RTB API. Second, a partner who wants to place an ad, would bid for it through an intermediary's Real Time Bidder, instead of building its own, and can access several RTB APIs. Third, a party who builds own real-time bidding platform which is mostly done by ad networks whose business model is based on targeting ads. It is also used by marketing agencies that retarget providers and customers as well [3] [13]. The first study presented in this paper explores if RTB has proven its effectiveness in Kuwait in terms of cookies collection relevance of displayed ads.

\section{The Platforms}

There are several Ad Exchange arenas that provide several types of platforms depending on the company's use of the platform. Some want to hire someone to do the whole process for them, bid and buy slots, others want to do the bidding through an intermediary and some want to build the whole thing and host it. Some Ad Agencies have access to several programmatic ad platforms that Google, AOL, Facebook, and many smaller firms make available to interested parties [8]. Google has three different RTB platforms Google Display Network, DoubleClick Ad Exchange and Invite Media; each is targeted for different users. First, the Google Display Network (GDN) is designed for advertising agencies to do real time buying of ad slots with full transparency, but the intermediary is GDN instead of buying directly from the publisher. Bidders can choose their audience based on demographics and geographical location as well as retargeting potential clients based on their surfing history. For example, someone enters a website to buy an item but does not proceed to check out. The website collects cookies and other data that reflect visitor's behavior, click pattern and interests. When the surfer visits any other website that placed their ad slots on the Google Display Network, advertisers are able to get this data and bid for the spot. 
They can then show the person the items relevant to what they searched for or the items that are exact matches to what they searched for, that is retargeting. The second platform provided by google is the DoubleClick Ad Exchange (ADX), this platform gives the option for the Ad Slot Buyer can directly buy the ad slot from the publisher directly. On this platform, advertisers bring their own data and strategy and based on that they can choose their optimal ad spot, in order to achieve their marketing goal. The third platform which was acquired by Google is Invite Media platform. Invite Media deals with high volume transactions and deals with the demand side, it also allows buyers to create bidding rules and optimizations. It connects bidders with several ad exchanges and suppliers [3].

\section{Our Study}

Instead of spending fortunes to advertise on the television, newspaper or radio stations, whose effectiveness and efficiency in reaching their targeted audience cannot be assessed easily, RTB seems to be a more attractive option. As this type of advertisements requires two sides, a supply side and a demand side, it cannot be fully accomplished on the company side. RTB has demanding technological requirements, a dedicated server to store the data and an information system that organizes the data in order for it to be useful. So, a company could usually be on one of the three sides, the supplier, intermediary or the bidder. The supplier could directly work with the bidder in a reservation agreement, but in order to start an auction they need to use a platform in which an algorithm is in use to make sure the advertisement slot goes to the highest bidder in less than a second. A company can build and host its own RTB API but this company would only specialize in providing the bidders with the supply of the publishers to auction. Using these Platforms to bid for advertisements, based on available data, consumers will eventually get advertisements relevant to their interests. Once the right person is reached with the right message at the right time, and clicking on the advertisement then the goal of programmatic advertising has been achieved. This is a step closer to efficient marketing. This can only happen if all the collected data is accurate. In order to know if the collection of cookies gets the right ads to the users, a two-step study is conducted here. The first step involves visiting several websites and ranking the ads that appear on those sites based on relevance. The second part is conducting a survey to assess if RTB is channeling to users ads that are relevant to their target product or service, and if it is acceptable or not. The objective of this study is to know if it is accurately working in Kuwait or not? And is there an opportunity in the Kuwaiti market or not.

\subsection{The First Part}

The first part of our investigation aims at assessing, after picking a target product on a specific website by a group of users, whether that product resembles any of those 
appearing in the ads on other subsequently visited websites or not. To carry out this part of the study, the following steps are followed

- Clear all cookies and history (to start fresh)

- Search for a certain product on one site (to test a target product)

- Search for the same product on a different other website using the same client computer or the same local server (to test the tracking accuracy)

- Visit ten other websites and count the number of ads on the opening page

- Count the number of ads of interest on the website, and calculate the percentage of those in comparison to the total ads displayed

- If the relevant ads constitute $50 \%$ or more, then the result would advocate the fact that RTB and programmatic advertisements do work in that they collect the right information. (relevant ads are ads that are related to the item searched for, or the exact same product.)

For example, among others considered by each user, a search for GoPro Hero 5 camera was done. First, the actual GoPro website was visited, and then a redirection is made to the website of the official provider of the product in Kuwait. Then, several sites that reviewed the GoPro were accessed. Other websites related to that product were also entered in order to give the chance for different websites to collect cookies. Most websites offered to access cookies in order to exploit those to show customized ads. After that, other different websites were accessed and the total number of ads displayed was counted. Specifically, the ads of interest were counted and then the percentage of those. Based on the results obtained, there were no ads relevant to the GoPro Hero 5 product seen in any of the websites accessed, but most ads were relevant to the surfer location instead. An example is a KLM ad that offers great deals from Kuwait to Amsterdam, or of no relevance at all like a university in America. The total number of ads spotted was 38, collected from 10 different websites of which only 8 ads were interesting, although they were not relevant to the searched product. Only 4 out of the 10 sites accessed scored more than $50 \%$ on the ads that were interesting to the user. This was repeated for several other products (a total of 45). The results did not change much. Hence, based on the results of this part of the study, RTB fails to a good extent to target the right audience. It must be kept in mind that this study was centered on Kuwait. The results of this first part are summarized in Table 1. 
Table 1. A measure of the relevance of displayed ads to users' interests.

\begin{tabular}{lcc}
\hline \multicolumn{1}{c}{ Website } & $\begin{array}{c}\text { Number of ad- } \\
\text { vertisements }\end{array}$ & Relevance \\
\hline investopedia.com & 4 & $0.00 \%$ \\
marketwatch.com & 1 & $0.00 \%$ \\
youtube.com & 1 & $100.00 \%$ \\
tweakandtrick.com & 2 & $50.00 \%$ \\
finance.yahoo.com & 1 & $0.00 \%$ \\
alwatan.kuwait.tt & 3 & $66.67 \%$ \\
electronics.howstuffworks.com & 5 & $0.00 \%$ \\
makeuseof.com & 13 & $23.08 \%$ \\
instructables.com & 6 & $16.67 \%$ \\
mirror.co.uk & 2 & $50.00 \%$ \\
Total & 38 & $21.05 \%$ \\
\hline
\end{tabular}

\subsection{The Second Phase}

The second component of the study is done in order to validate the results obtained from the first component of the investigation. It uses a survey of nine questions. Seventy-two responses were collected. The survey details are presented in the Appendix. Females constituted $65 \%$ of the respondents, and $55 \%$ of the respondents were between the age of 18 and 27. A percentage of $49 \%$ of the respondents spend 4-7 hours online. Of the 72 responses, only $11 \%$ always shop online, while $40 \%$ rarely do so. Having this data, classifies the respondents as frequent internet users, and their age is the most targeted as they are the trendsetters, looking at this data it is expected that the cookies collected from them are valuable and most will bid for this market, depending on what they are looking for as well. Most of the respondents claim that they see 1-10 ads online, yet only $7 \%$ of the respondents claim that the ads are always 
relevant, while $40 \%$ answered rarely. $70 \%$ of the respondents answered that they do not click on any ad whether it is of interest to them or not. $15 \%$ of the respondents actually bought something as a result of seeing an online ad. Also, $36 \%$ of the respondents answered that yes, they do get inspired to buy products online. Thus, this shows that less people click on ads and buy anything through clicking such ads. This is done mainly to escape spam material. It can also be deducted that there is a security issue, where the ad viewers would be skeptical to click on ads directly fearing spyware infiltration or getting pop-up ads that would slow down the network. Kuwait's conservative culture refrains users from allowing cookies to be collected from them, or stored on their computer.

\subsection{Findings and Analysis}

Our findings reveal that most users do not find any of the ads relevant to their needs. This probably suggests that either the collection of cookies is not sufficient to do efficient RTB and hence show the right ads. A better platform that forms a promising vehicle for RTB is social networking. Consumers in Kuwait and in many other places use those extensively and a proper RTB needs to exploit the big data such platforms offer. The findings also suggest that the audience in Kuwait would be reluctant to click on the ads even if they were relevant or of interest to them, so the use of programmatic advertising is not very effective in Kuwait. Reasons may be attributed to the fact that although Programmatic Bidding is the closest thing to efficient marketing, means to increase its effectiveness and efficiency are certainly a subject of research. Those problems might be bad for the branding of a company. In principle, advertising activities either support the brand image or can be performance-related [9]. If a company's ads end up on inappropriate web site ad slots, the brand image of the company is negatively influenced. Bidders may not be provided with a full profile of the assortment of items hosted in the unit [7]. Transparency is a key issue here. Another problem that might be unintendedly caused is placing an ad in a ghost website and paying for an ad slot that might never be seen. As it is very expensive to build and host an RTB platform, it is very crucial to choose the correct ad exchange network that is most transparent, fast, has a big database and multiple suppliers. Choosing an ideal ad exchange for your business model, will insure that your ads are being placed on real website, and seen by the target audience will not only cut costs paid for traditional advertising but also generate a higher revenue.

Our investigation reveals that some companies in Kuwait do use RTB as an advertising technique but there is no Real-Time Bidding API based and built in Kuwait. Most of the ads that appear on Kuwaiti websites are for locations and business in the region mostly Qatar and Dubai. Technology in Kuwait is not as advanced as it is in other parts of the world like the United States, Europe or India. There is hardly any IT Solution building companies established or built in Kuwait, they are mostly foreign and outsourced. There are two approaches that might be seen in the future in Kuwait. The first approach avoids building own platforms for RTB as this is time consuming and expensive. Instead, it might be more beneficial in Kuwait to utilize RTB API's built by companies like Google, Yahoo or Facebook. The full potentials of the RTB 
approach need be understood in order for the motivation and the investment to occur. The second approach is to establish an RTB API in Kuwait that does not only serve Kuwait only but also serves the MENA region with joint MENA investment. The second approach seems to be currently farfetched as it requires Kuwait to mature technologically in order for this to succeed. Given the above, we believe that users in Kuwait will realize the full potential of RTB once relevance of displayed ads is achieved to a great extent. This also encourages proper utilization of the technique by businesses with online presence in the country to ensure the consumers are targeted with relevant ad material and hence obtain an attractive Return on Investment.

\section{Conclusion}

Programmatic Advertising (especially RTB) aims at cutting advertising costs and generates higher revenue by achieving efficient marketing. It is apparent that efficient marketing is the utopia of marketing and cannot be fully achieved. Having access to data by using cookies is a closer step to marketing to the right person at the right time. Newer technologies that collect user information might prove very useful in realizing the full potential of RTB. Social networking platforms, a source of big data, present a rich resource for RTB that need to be utilized to its fullest extent. There are several kinds of platforms to do RTB. So, using RTB platforms wisely and choosing the right platform is crucial to the business marketing plan. In Kuwait, RTB is not widely used as the infrastructure needs to evolve to acquire this technology and thus the data. This would provide businesses with enough encouragement to utilize RTB and thus gain the technology's full potential of offering efficient educated targeted marketing. The

Kuwaiti culture is tight, so marketing techniques mainly used in Kuwait is the word of mouth, people usually trust the experiences of other people around them. Hence, it would also be an opportunity to use clips with famous social influencers, to advertise for a certain product. Doing so, the Kuwaiti market would start to trust the adverts they see online.

\section{References}

1. Berke, A., State of performance marketing 2017: North America \& Europe. State Of Performance Marketing. Adroll, https://www.adroll.com/assets/pdfs/guides-andreports/adroll-state-of-performance-marketing-17.pdf, (2017).

2. Fernandez-Tapia, J., Guéant, O. and Lasry, J., Optimal Real-Time Bidding Strategies. Applied Mathematics Research eXpress, (2016).

3. Google Inc., The Arrival of Real-Time Bidding and What it Means for Media Buyers. White Paper Review (2011).

4. Grether, M., Using Big Data for Online Advertising without Wastage: Wishful Dream, Nightmare or Reality?. Gfk-Marketing Intelligence Review , 8(2), 38-42 (2016).

5. Moghrabi, I.A.R. and Al-Mohammad, A., Social Media or Social Business Networks", Lecture Notes in Computer Science, 9844, IE3, 42-52 (2016).

6. Münstermann, H. and Würtenberger, P., Programmatic Disruption for Premium Publishers. In Programmatic Advertising, Springer International Publishing, Netherland (2016). 
7. Sayedi, A., 2017. Real-Time Bidding in Online Display Advertising, Washington: University of Washington.

8. Sun, Z. e. a.,. The Making of a Good Impression: Information Hiding in Ad Exchanges. MIS Quarterly, 40(3), 717-724 (2016).

9. Traver, C. G. \& Laudon, K. C., E-comemrce 2015: business. technology. society. 11th ed., Pearson (2015).

10. Vickrey, W., Counterspeculation, auctions, and competitive sealed tenders. The Journal of finance, 16(1), 8-37 (1961)..

11. Yuan, S. Supply Side Optimisation in Online Display Advertising (Doctoral dissertation, UCL (University College London)) (2015).

12. Yuan, S., \& Wang, J., Sequential selection of correlated ads by POMDPs. In Proceedings of the 21st ACM international conference on Information and knowledge management, 515-524 (2012)..

13. Zhang, W., Yuan, S., \& Wang, J., Optimal real-time bidding for display advertising. In Proceedings of the 20th ACM SIGKDD international conference on Knowledge discovery and data mining, 1077-1086 (2014).. 


\section{Appendix}

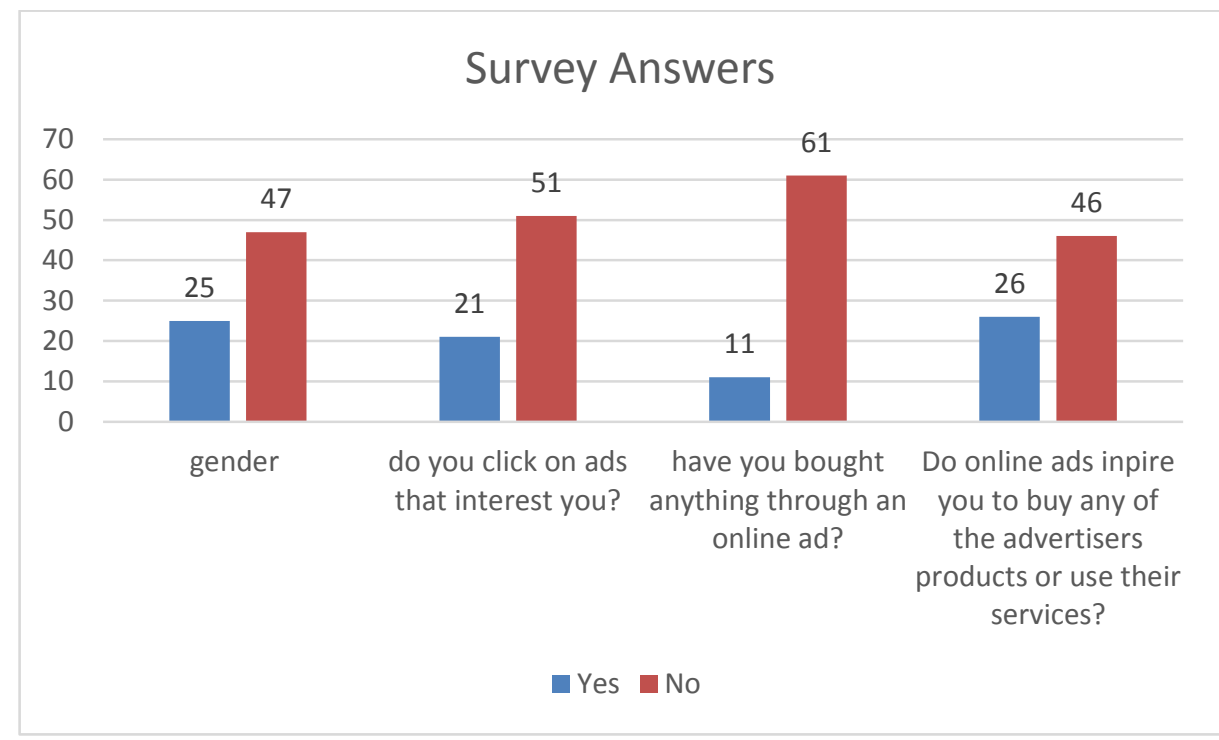

Please note that in the gender data, Red is Female and Blue is Male.

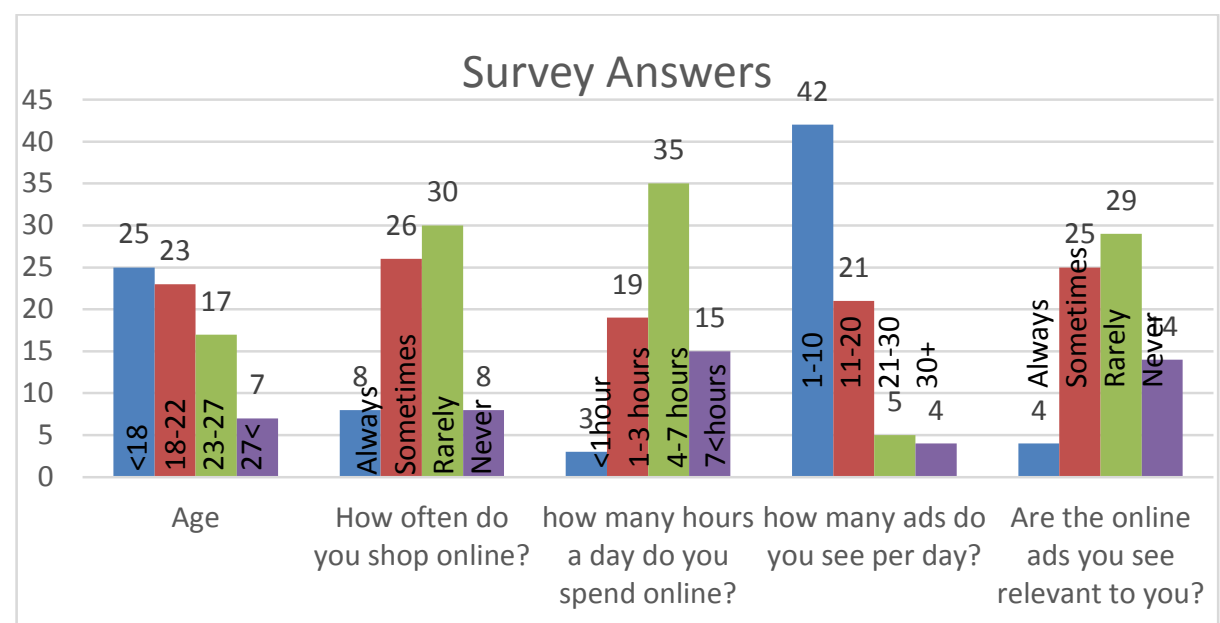

